Revista Chilena
DE Pediatría

www.revistachilenadepediatria.cl

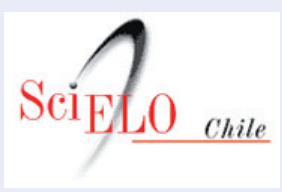

www.scielo.cl

Rev Chil Pediatr. 2020;91(6):995-996

DOI: $10.32641 /$ rchped.v91i6.3370

CARTA AL EDITOR

\section{La inferencia bayesiana como replicación y cuantificación en la investigación clínica}

Bayesian inference as replication and quantification in clinical research

\section{Sr. Editor,}

En un estudio reciente de la presente revista se refiere que la metodología estadística bayesiana es relativamente novedosa y que se viene aplicando cada vez más en la investigación científica ${ }^{1}$ lo cual ha permitido aportar una renovación importante en el análisis de los datos complementado la estadística clásica.

Esta carta tiene como fin presentar un ejemplo sencillo de inferencia bayesiana a partir de los datos del artículo de Soto y otros ${ }^{2}$ que reporta una correlación de Pearson significativa entre la autoeficacia (AU) y calidad de vida (CV) en pacientes con cardiopatía congénita mediante el test de significancia de la hipótesis nula (NHST, por sus siglas en ingles) de mayor uso en investigaciones clínicas.

La estadística bayesiana permite cuantificar la evidencia asociada a las hipótesis y a sus modelos asociados, lo que refuerza la metodología estadística que permite estimar de forma más contundente, por ejemplo, el rechazo de la hipótesis nula en análisis correlaciónales $^{3}$. Este enfoque utiliza el factor Bayes como método cuantificable del grado en que los datos respaldan a dos modelos que pueden nombrarse clásicamente como la hipótesis nula o a la hipótesis alterna, y cuyos valores interpretables ${ }^{3,4,5}$ son sistematizados en: "débil", "moderado", "fuerte" y "muy fuerte" (tabla 1).

Para el fin de la presente carta se tuvo en cuenta el tamaño de la muestra $(n=51)$ y el valor de correlación ${ }^{4}$ de AU-CV $(r=0,342)$ reportado por Soto y otros ${ }^{2}$ lo cual permite estimar la credibilidad de las hipótesis estadísticas de acuerdo a la muestra de estudio. ${ }^{4}$

El factor Bayes permite inferir dos interpretaciones: $\mathrm{FB}_{10}$ (a favor de la hipótesis alternativa de significancia) y $\mathrm{BF}_{01}$ (a favor de la hipótesis nula) y el intervalo de cre- dibilidad al 95\%. Los resultados obtenidos del factor Bayes son: $\mathrm{BF}_{10}=3,281$ y $\mathrm{BF}_{01}=0,305$ e IC95\% [0,069 a 0,553]. Este modelo bayesiano señaló una magnitud de evidencia moderada hacia la hipótesis de correlación de AU-CV lo cual fortalece los resultados de Soto y otros ${ }^{2}$ pues respaldan con una evidencia moderada a favor de la hipótesis alterna unas 3 veces mayor a algún posible hallazgo de nulidad. Asimismo, también se reporta el parámetro del factor Bayes máximo $\left(\operatorname{maxBF}_{10}=5,091\right)$ cuyo valor similar de respaldo moderado permite determinar la estabilidad de los hallazgos ${ }^{3}$.

Por lo tanto, la inclusión del factor Bayes sería un gran aporte para el análisis probabilístico inferencial en el planteamiento de las hipótesis estadísticas, además permite la reevaluación de los resultados de investigaciones reales anteriores para determinar la certeza de sus conclusiones en análisis correlacionales incluso cuando no se cuenta con acceso a los datos. Esto es de gran relevancia también en las investigaciones sistemáticas de metaanálisis y otras que incluyan medidas que se basan en la metodología $\mathrm{NHST}^{1,4}$ para el contraste inclusivo de las hipótesis estadísticas.

Tabla 1. Valores de interpretación cuantificable del factor Bayes

\begin{tabular}{lll}
\hline$>30$ & Muy fuerte & Hipótesis alternativa \\
$10+30$ & Fuerte & Hipótesis alternativa \\
$3,1-10$ & Moderado & Hipótesis alternativa \\
$1,1-3$ & Débil & Hipótesis alternativa \\
1 & 0 & No evidencia \\
$0,3-0,9$ & Débil & Hipótesis nula \\
$0,29-0,1$ & Moderado & Hipótesis nula \\
$0,09-0,03$ & Fuerte & Hipótesis nula \\
$<0,03$ & Muy fuerte & Hipótesis nula \\
\hline
\end{tabular}

Nota: Creación propia según Jefreys ${ }^{5}$

Cristian Antony Ramos-Vera

Universidad Cesar Vallejo.

Sociedad Peruana de Psicometría. Perú.

Correspondencia:

Cristian Antony Ramos-Vera

cristony_777@hotmail.com 


\section{Referencias}

1. Cáceres Guido P, Humberto Pavan C, Otamendi E, Bramuglia F. Principios de estadística Bayesiana y su relación con la farmacocinética aplicada. Rev Chil Pediatr. 2020;91(5):1-10. http://dx.doi.org/10.32641/rchped.vi91i5.1594.

2. Soto M, Zubarew T, Arancibia-Galilea MF. Adolescentes y jóvenes portadores de cardiopatías congénitas en etapa de transferencia a la atención médica de adultos. Rev Chil Pediatr. 2020;91(3):339-46. http://dx.doi.org/10.32641/ rchped.v91i3.1346
3. Nuzzo RL. An introduction to Bayesian data analysis for correlations. PM\&R. 2017;9(12):1278-82. https://doi. org/10.1016/j.pmrj.2017.11.003.

4. Ly A, Raj A, Etz A, Gronau QF, Wagenmakers EJ. Bayesian reanalyses from summary statistics: a guide for academic consumers. Adv Meth Pract Psychol Sci. 2018;(3):367-74.

Disponible en: https://journals.sagepub.com/doi/ full/10.1177/2515245918779348.

5. Jeffreys H. Theory of probability (3rd ed.). New York: Oxford University Press. 1961. 